\title{
Relaxation of ${ }^{27} \mathrm{Al}$ NMR in Aluminium Tribromide due to Raman Process
}

\author{
Noriaki Okubo ${ }^{a}$, Mutsuo Igarashi ${ }^{b}$, and Ryozo Yoshizaki ${ }^{c}$ \\ a Institute of Physics, University of Tsukuba, Tsukuba 305, Japan \\ b Department of Applied Physics, Gunma College of Technology, Maebashi 371, Japan \\ c Institute of Applied Physics, University of Tsukuba, Tsukuba 305, Japan
}

Z. Naturforsch. 51a, 277-282 (1996); received November 15, 1995

\begin{abstract}
The nuclear spin-lattice relaxation of ${ }^{27} \mathrm{Al} \mathrm{NMR}$ in $\mathrm{AlBr}_{3}$ has been examined from 25 to $320 \mathrm{~K}$. The result is analyzed using a theory of the Raman process based on covalency. A Debye temperature of $118 \mathrm{~K}$ is obtained together with a covalency larger than that obtained from the relaxation in the ${ }^{81} \mathrm{Br}$ NQR.

Key words: Aluminium tribromide; ${ }^{27} \mathrm{Al}$ NMR; Nuclear quadrupole relaxation; Raman process; Covalency.
\end{abstract}

\section{Introduction}

In the preceding paper [1] we dealt with the nuclear relaxation of bromine NQR in aluminium tribromide $\left(\mathrm{AlBr}_{3}\right)$ using a theory of the Raman process based on covalency [2]. Although the aluminium NQR is also reported in [3-6], there are some difficulties in applying the theory to the relaxation [1]. One of the difficulties originates from the large asymmetry parameter 0.747 [4]. This brings about considerable mixing among the states specified by the magnetic quantum number $m$. Then the transition probabilities for the quadrupolar relaxation involve large cross-terms of the matrix elements for transitions $\Delta m= \pm 1$ and $\Delta m= \pm 2$, and the existing theories cannot be applied. When the relaxation is treated in a strong external magnetic field, this problem is avoided since the Hamiltonian is then dominated by the Zeeman term; the eigen states can be specified properly by $m$. The purpose of the present paper is to show a way of handling the remaining difficulties and to provide information complementary to the bromine NQR study.

There is another motivation for the present study. $\mathrm{AlBr}_{3}$ molecules are known to intercalate into graphite with $\mathrm{Br}_{2}$ molecules to form ternary compounds [7]. We found that the ${ }^{27} \mathrm{Al} \mathrm{NMR}$ of $\mathrm{AlBr}_{3}$ changes drastically on intercalation [8]. However, there is little information about the relaxation in pure $\mathrm{AlBr}_{3}$. To ob-

Reprint requests to Dr. N. Okubo. tain the standard for comparison of the NMR is the second purpose.

\section{Experimental}

Since $\mathrm{AlBr}_{3}$ is extremely hygroscopic, it is difficult to obtain ideal powder samples. The sample was prepared by melting and annealing as before, but a quartz ampoule was used in place of the pyrex one to avoid the ${ }^{27} \mathrm{Al}$ signal from pyrex glass.

The use of NMR for observing the ${ }^{27} \mathrm{Al}$ nuclei has an advantage over NQR: The resonance frequency is elevated up to a more reasonable value, and the observation is facilitated. The ${ }^{27} \mathrm{Al} \mathrm{NMR}$ was observed with a pulse spectrometer (Matec Inc.). The relaxation was examined from $25 \mathrm{~K}$ to $320 \mathrm{~K}$ in a field of $7 \mathrm{~T}$ but also in $4.7 \mathrm{~T}$ at $77 \mathrm{~K}$ and room temperature. The spin echo signal after $\pi / 2-\pi$ pulses was monitored. The spectrum was obtained as the envelope of the echo height measured with varying the frequency. The relaxation was examined by measuring the echo height at the center frequency, $\mathrm{S}(\mathrm{t})$, at time $t$ after saturation by another $\pi / 2$ pulse. $T_{2}$ was $2.5 \pm 0.5 \mathrm{~ms}$ and $T_{2}^{*}$ was $16 \pm 4 \mu$ s, typically at $77 \mathrm{~K}$.

\section{Results and Discussion}

\subsection{Origin of Relaxation}

Figure 1 shows the line shape of ${ }^{27} \mathrm{Al}$ NMR. The pattern was almost independent of the temperature, 


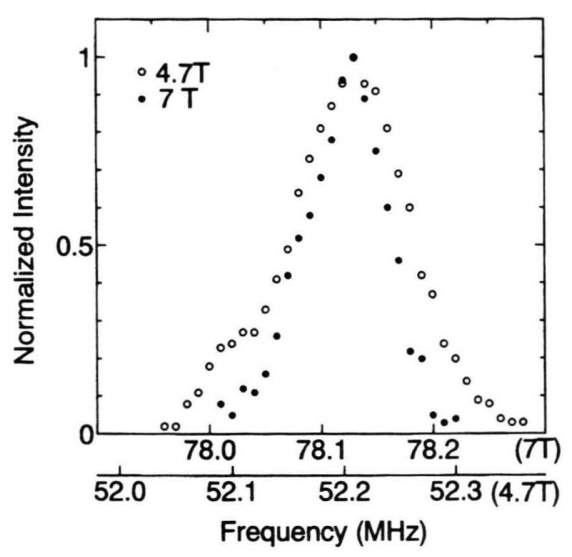

Fig. 1. The line shape of ${ }^{27} \mathrm{Al} \mathrm{NMR}$ in $\mathrm{AlBr}_{3}$ at room temperature. The pattern for $7 \mathrm{~T}$ is so shifted that the peak is superposed on that for $4.7 \mathrm{~T}$.

but somewhat dependent on the setting of the sample in the magnet because it was polycrystalline. However, it roughly corresponds to the powder pattern [9] derived with the coupling constant $e^{2} Q q=13.57 \mathrm{MHz}$ and the asymmetry parameter $\eta=0.747$ [4], and the width is nearly inversely proportional to the strength of the field. Therefore the observed signal is the central transition $(m=1 / 2$ to $-1 / 2)$ broadened mainly by the second-order perturbation of the quadrupolar interaction.

The magnetic relaxation of the system with spin $I=5 / 2$ is governed by three characteristic times, under an initial condition symmetrical about $\pm m$. They are written as $(2 W)^{-1},(12 W)^{-1}$, and $(30 W)^{-1}$, when the transition probability from the level $m$ to $m+1$, $P(m, m+1)$, is written as $W(I+1)(I-m+1)[10]$. On the other hand, the quadrupolar relaxation is governed by three characteristic times, which are the inverse of the roots of the following secular equation [10]:

$$
\begin{aligned}
100 \lambda^{3} & +280 \lambda^{2}\left(W_{1}+W_{2}\right) \\
& +3 \lambda\left(40 W_{1}^{2}+208 W_{1} W_{2}+45 W_{2}^{2}\right) \\
& +36 W_{1} W_{2}\left(4 W_{1}+7 W_{2}\right)=0,
\end{aligned}
$$

where $W_{1}$ and $W_{2}$ are defined with $P(m, m+\mu)$ $(\mu=1,2)$ as

$P(m, m+1)=\frac{(2 m+1)^{2}(I-m)(I+m+1)}{2 I(2 I-1)^{2}} W_{1}$,

$P(m, m+2)=\frac{(I-m-1)(I+m+2)(I-m)(I+m+1)}{2 I(2 I-1)^{2}} W_{2}$.
The magnetization recovery curve can be derived by regarding the effect of application of saturating $\pi / 2$ pulse as only equalizing the populations of two levels corresponding to the frequency. For the magnetic relaxation of the system it can be shown that

$$
\begin{aligned}
{[S(\infty)-S(t)] / S(\infty) } & =\frac{1}{35} \exp (-2 W t) \\
& +\frac{8}{45} \exp (-12 W t)+\frac{50}{63} \exp (-30 W t) .
\end{aligned}
$$

On the other hand, for the quadrupolar relaxation the pre-exponential coefficients depend on the ratio of $W_{1}$ and $W_{2}$ but, as shown later, we are particularly concerned with the case $W_{1}=W_{2}(\equiv W)$. Then

$$
\begin{aligned}
{[S(\infty)} & -S(t)] / S(\infty)=\frac{1}{35} \exp \left(-\frac{4}{5} W t\right) \\
& +\frac{50}{63} \exp \left(-\frac{3}{2} W t\right)+\frac{8}{45} \exp \left(-\frac{33}{10} W t\right) .
\end{aligned}
$$

When the observed recovery curves are fitted by both equations, a clear difference appears only at values of $[S(\infty)-S(t)] / S(\infty)$ smaller than $10^{-1}$. An example is shown in Figure 2. The signal-to-noise ratio was not sufficient to discriminate the two relaxation mechanisms by this method. However, when the recovery curves for all temperatures are fitted by (4), $W$ shows $T^{2}$ dependence at high temperatures, as shown in Figure 3. This suggests that the relaxation is governed by the Raman process.

In the region including $77 \mathrm{~K}, W$ increased as the temperature increased, and the relaxation was almost independent of the strength of magnetic field at $77 \mathrm{~K}$.

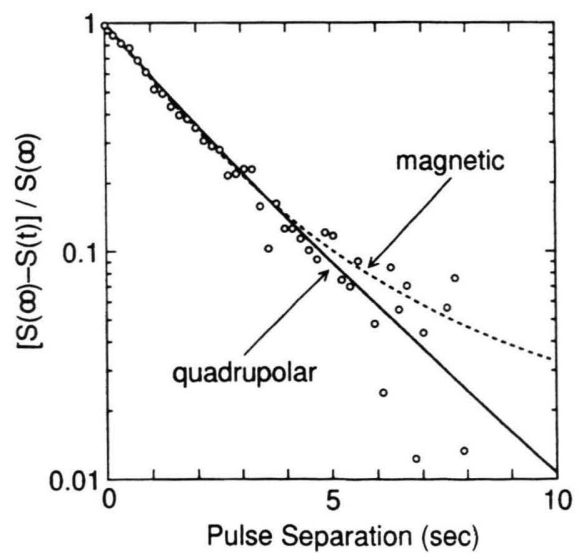

Fig. 2. An example of the magnetization recovery curve at $210 \mathrm{~K}$ in the field of $7 \mathrm{~T}$. 


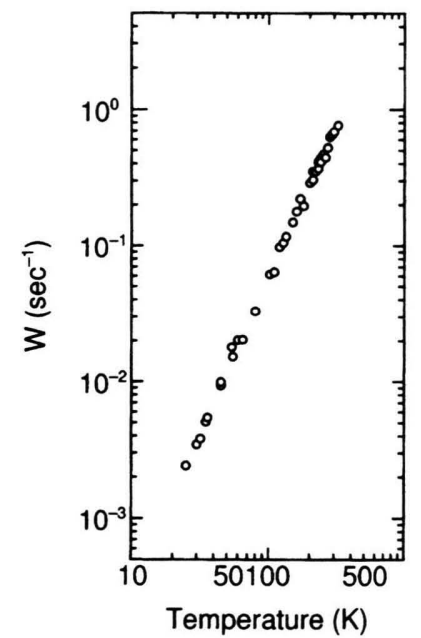

Fig. 3. Temperature dependence of the transition probability $W$ of the ${ }^{27} \mathrm{Al}-\mathrm{NMR}$ line in $\mathrm{AlBr}_{3}$.

If the BPP type relaxation mechanism was dominant, the relaxation rate should be inversely proportional to the square of the strength of the magnetic field in the region where it increases with increasing temperature, whether the relaxation is magnetic or quadrupolar. Therefore, the BPP type mechanism is excluded.

\subsection{Expression for Raman Process}

In the following the Raman process is assumed. For brevity, in the dimer $\mathrm{Al}_{2} \mathrm{Br}_{6}$ [11], three mirror planes perpendicular to each other are assumed; the bisecting plane of the terminal bonds contains the bridging bonds, and vice versa. The bridging $\mathrm{Br}$ atoms are numbered as 1 and 2 , and the terminal ones as 3 and 4, in accordance to [12]. Furthermore, in order to apply Yosida and Moriya's theory assuming the equivalence of the ions [2], the four $\mathrm{Br}$ ions are assumed to be equivalent, so that we do not expect complete numerical agreement between the theory and the experiment. Then $P(m, m+\mu)(\mu=1,2)$ due to the Raman process is given for any spin I by

$$
\begin{gathered}
P(m, m+\mu)=\frac{A^{\prime 2} c^{3}\left|Q_{\mu m}\right|^{2}}{2 \pi^{3} a^{7} d^{2} v_{\mathrm{s}}^{3}} \\
\cdot T^{* 2} \sum_{\phi} \sum_{v=1}^{3} N_{\mu v}(\phi) D_{v}\left(T^{*}, \phi\right), \quad(5) \\
A^{\prime}=\frac{3 e^{2} Q\left\langle r^{-3}\right\rangle}{10 I(2 I-1)}, \quad Q_{\mu m}=\left\langle m+\mu\left|Q_{\mu}\right| m\right\rangle, \\
Q_{ \pm 1}=\frac{1}{2}\left(I_{ \pm} I_{z}+I_{z} I_{ \pm}\right), \quad Q_{ \pm 2}=I_{ \pm}^{2} .
\end{gathered}
$$

The notation is the same as in [1]. In the present case, $\langle>$ means the expectation value with respect to the valence $p$ electron of $\mathrm{Al}$ atom. The sound velocity $v_{\mathrm{s}}$ is related to the Debye temperature $\theta_{\mathrm{D}}$ by

$$
\hbar \omega_{\mathrm{D}}=\hbar v_{\mathrm{s}} k_{\mathrm{D}}=k_{\mathrm{B}} \theta_{\mathrm{D}} .
$$

Summation over the angle made by any bond pair, $\phi$, has been introduced to avoid the use of large $v$ in applying the theory to the case involving several different bond angles, and instead $N_{\mu v}$ and $D_{v}(v=1$ to 3$)$ have been left to be functions of $\phi$.

Since the sample was, though not powder, polycrystalline, $N_{\mu \nu}$ must be averaged about the orientation of the field $\boldsymbol{H}_{0}$. After averaging, $N_{\mu v}$ is expressed with a measure of covalency, $\lambda$, and its first and second derivatives with respect to $r, \lambda^{\prime}$ and $\lambda^{\prime \prime}$, as follows:

$$
\begin{aligned}
N_{11}= & N_{12}=N_{13} \\
= & 8\left[\frac{1}{15}\left(77 \cos ^{2} \phi+1\right) u^{2}+\frac{1}{15}\left(3 \cos ^{2} \phi-1\right) v^{2}\right. \\
& \left.+\lambda^{2}+\frac{2}{15}\left(11 \cos ^{2} \phi-1\right) u v+\frac{8}{3} \lambda u+\frac{4}{15} \lambda v\right], \\
N_{21}= & N_{22}=N_{23}=N_{11} / 4,
\end{aligned}
$$

where

$$
u=\frac{1}{2} a \lambda^{\prime}-\lambda, \quad v=4 \lambda-\frac{5}{2} a \lambda^{\prime}+\frac{1}{2} a^{2} \lambda^{\prime \prime} .
$$

Equation (7) can be applied to any equivalent bond pair in NMR of powders. The derivation of these expressions is given in the Appendix.

$D_{v}\left(T^{*}\right)$ is defined as

$$
D_{v}\left(T^{*}\right)=T^{*} \int_{0}^{1 / T^{*}} \frac{x^{2} e^{x}}{\left(e^{x}-1\right)^{2}} L_{v}\left(c T^{*} x\right) \mathrm{d} x,
$$

where $x=\hbar \omega / k_{\mathrm{B}} T, \omega$ being the angular frequency of the phonons. $L_{\mathrm{v}}\left(c T^{*} x\right)=L_{\mathrm{v}}(k a)$ has generally the following forms:

$$
\begin{gathered}
L_{1}(k a)=\left\{S_{1} S_{2}\right\}_{\boldsymbol{k}}^{2}=\left[-\frac{1}{2} f(\sqrt{2(1+\cos \phi)} k a)\right. \\
\left.+\frac{1}{2} f(\sqrt{2(1-\cos \phi)} k a)\right]^{2}, \\
\begin{aligned}
& L_{2}(k a)=\left\{C_{1} C_{2}\right\}_{\boldsymbol{k}}^{2}= {[1-2 f(k a)} \\
&+\frac{1}{2} f(\sqrt{2(1+\cos \phi)} k a) \\
&\left.+\frac{1}{2} f(\sqrt{2(1-\cos \phi)} k a)\right]^{2}, \\
& L_{3}(k a)=2\left\{S_{1} S_{2} C_{1} C_{2}\right\}_{\boldsymbol{k}} \\
&=2\left[-\frac{1}{2} f(\sqrt{2(1+\cos \phi)} k a)\right. \\
&\left.+\frac{1}{2} f(\sqrt{2(1-\cos \phi)} k a)\right] \\
& \cdot\left[1-2 f(k a)+\frac{1}{2} f(\sqrt{2(1+\cos \phi)} k a)\right. \\
&\left.\quad+\frac{1}{2} f(\sqrt{2(1-\cos \phi)} k a)\right],
\end{aligned}
\end{gathered}
$$


where

$$
\begin{aligned}
& S_{n}=\sin (a \boldsymbol{k} \cdot \boldsymbol{n}), \quad C_{n}=\cos (a \boldsymbol{k} \cdot \boldsymbol{n})-1, \\
& f(y)=\frac{\sin y}{y}
\end{aligned}
$$

and \{\}$_{\boldsymbol{k}}$ means the average about the direction of $\boldsymbol{k}$, $\boldsymbol{k}$ being the wave vector of the phonon, and $k$ its magnitude. $\boldsymbol{n}$ denotes the unit vector along the bond.

Since $W_{\mu}(\mu=1,2)$ is related with $P(m, m+\mu)$ by (2) and $N_{2 v}=N_{1 v} / 4$ by (7), we obtain $W_{1}=W_{2}=W$. This equality holds for any value of spin. $W$ for $I=5 / 2$ is then written as

$$
W=\frac{9 e^{4} Q^{2}\left\langle r^{-3}\right\rangle^{2} c^{3}}{1000 \pi^{3} a^{7} d^{2} v_{\mathrm{s}}^{3}} T^{* 2} \sum_{\phi} \sum_{v=1}^{3} N_{1 v}(\phi) D_{v}\left(T^{*}, \phi\right) .
$$

\subsection{Estimation of Debye Temperature}

Introducing a scaling time $\tau$ defined as

$$
\tau^{-1}=\frac{9 e^{4} Q^{2}\left\langle r^{-3}\right\rangle_{\mathrm{A} 1}^{2} c^{3}}{1000 \pi^{3} a^{7} d^{2} v_{\mathrm{s}}^{3}} N_{11}(0),
$$

(10) can be reduced to a form convenient for fitting: When bridging bonds are neglected,

$$
\begin{aligned}
W T^{-2}=\left(\tau \theta_{\mathrm{D}}^{2}\right)^{-1} & {\left[\sum_{v=1}^{3} D_{v}\left(T^{*}, 0\right)\right.} \\
& \left.+\varepsilon\left(\phi_{34}\right) \sum_{v=1}^{3} D_{v}\left(T^{*}, \phi_{34}\right)\right],
\end{aligned}
$$

and when bridging bonds are also taken into account,

$$
\begin{aligned}
W T^{-2}=\left(\tau \theta_{\mathrm{D}}^{2}\right)^{-1}[ & 2 \sum_{\mathrm{v}=1}^{3} D_{\mathrm{v}}\left(T^{*}, 0\right) \\
& +\varepsilon\left(\phi_{12}\right) \sum_{\mathrm{v}=1}^{3} D_{v}\left(T^{*}, \phi_{12}\right) \\
& +\varepsilon\left(\phi_{34}\right) \sum_{\mathrm{v}=1}^{3} D_{\mathrm{v}}\left(T^{*}, \phi_{34}\right) \\
& \left.+4 \varepsilon\left(\phi_{13}\right) \sum_{\mathrm{v}=1}^{3} D_{\mathrm{v}}\left(T^{*}, \phi_{13}\right)\right] .
\end{aligned}
$$

In (12) and (13), $\phi_{n, n^{\prime}}$ denotes the angle between the bonds $\boldsymbol{n}$ and $\boldsymbol{n}^{\prime}$, and

$$
\varepsilon\left(\phi_{n, n^{\prime}}\right)=\frac{N_{11}\left(\phi_{n, n^{\prime}}\right)}{N_{11}(0)} .
$$

By fitting (12) or (13) to the experimental result, we can determine $\theta_{\mathbf{D}}$ together with $\tau$ as fitting parameters. The relation

$$
\lambda \propto \exp (-r / \varrho)
$$

Table 1. Values of $N_{1 v}(v=1$ to 3$)$ for the bond pair making the angle $\phi$, in unit of $\lambda^{2}$. The value of $\phi_{13}$ was calculated from the values of $\phi_{12}$ and $\phi_{34}$ [11], assuming three mirror planes in $\mathrm{Al}_{2} \mathrm{Br}_{6}$ dimer.

\begin{tabular}{lllll}
\hline$\phi$ & $\begin{array}{l}\phi_{n n} \\
\left(=0^{\circ}\right)\end{array}$ & $\begin{array}{l}\phi_{12} \\
\left(=97.9^{\circ}\right)\end{array}$ & $\begin{array}{l}\phi_{34} \\
\left(=114.7^{\circ}\right)\end{array}$ & $\begin{array}{l}\phi_{13} \\
\left(=110.8^{\circ}\right)\end{array}$ \\
\hline$N_{1 v}(\phi)$ & 746.4 & 167.8 & -109.4 & -37.9 \\
\hline
\end{tabular}

Table 2. Debye temperature $\theta_{\mathrm{D}}$, scaling time $\tau$, and a measure of covalency $\lambda$.

\begin{tabular}{llrl}
\hline & $\theta_{\mathrm{D}}(\mathrm{K})$ & \multicolumn{1}{c}{$\tau(\mathrm{s})$} & \multicolumn{1}{l}{$\lambda$} \\
\hline Bridging neglected & 117.8 & 7.41 & 0.150 \\
Bridging included & 117.6 & 15.15 & 0.104 \\
\hline
\end{tabular}

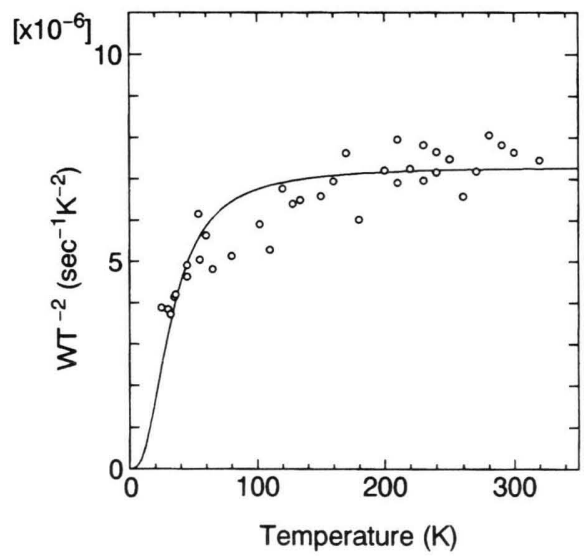

Fig. 4. The result of a least-squares-fitting of (12). The result for (13) is omitted because it is almost superposed by that for (12).

was assumed with a value of $0.345 \AA$ for the repulsive range parameter $\varrho$ as before [1, 2]. Using $a=2.28 \AA$ as an average for terminal bonds we have $u=-4.304 \lambda$ and $v=42.359 \lambda$. The values of $N_{1 v}$ calculated from (7) are given in Table 1. A value of 2.753 was used for $c=k_{\mathrm{D}} a$. The integration in (8) was carried out numerically. Table 2 and Fig. 4 show the results of a leastsquare-fitting. The present value of $\theta_{\mathrm{D}}$ for $\mathrm{Al}$ atoms, 117.8 or $117.6 \mathrm{~K}$, is much higher than that obtained for $\mathrm{Br}$ atoms, $67.6 \mathrm{~K}$ [1]. For $\mathrm{AlBr}_{3}$ the value of $\theta_{\mathrm{D}}$ has not been determined either from the experiment of the specific heat or from the temperature factor in the $\mathrm{X}$-ray analysis. In the case of $\mathrm{SbCl}_{3}$ [13] a value of $\theta_{\mathbf{D}}$ higher than that for the halogen atoms was calculated for the central metal from the temperature factor. For 
$v_{\mathrm{s}}$ we obtain a value of 1.30 or $1.29 \times 10^{5} \mathrm{~cm} / \mathrm{sec}$ by means of [6].

\subsection{Estimation of Covalency}

Now we can estimate $\lambda$ from $\tau$ using (11). The $e^{2} Q\left\langle r^{-3}\right\rangle$ is given by (5/4) $e^{2} Q q_{\mathrm{at}}, e^{2} Q q_{\mathrm{at}}$ being the quadrupole coupling constant for the free atom, reported to be $37.52 \mathrm{MHz}$ for ${ }^{27} \mathrm{Al}$ [14]. We obtained the values of $\lambda$ in Table 2 . $\lambda$ corresponds to the number of electrons on each bond, effective for the largest component of the EFG along the bond [1,13]. Therefore, when there are several bonds, $\lambda$ should be compared not with the ratio $f=e^{2} Q q_{\mathrm{mol}} / e^{2} Q q_{\mathrm{at}}$ for the whole molecule buth with $f$ for each bond.

Casabella et al. [4] analyzed the ${ }^{27} \mathrm{Al}$ NQR frequency with the expression

$\frac{e^{2} Q q_{\mathrm{mol}}}{e^{2} Q q_{\mathrm{at}}}=\frac{\left(A_{\mathrm{M}}-B_{\mathrm{M}}\right)}{2}\left[1+\cot ^{2}\left(\phi_{12} / 2\right)\right]\left(1+\varrho_{\mathrm{M}} \varepsilon\right)$,

where $A_{\mathrm{M}}$ denotes the electron population of each terminal orbital of the $\mathrm{Al}$ atom and $B_{\mathrm{M}}$ that of the bridging one. From the Br NQR frequency they determined the excess of electrons on $\mathrm{Br}$ atoms and then estimated the net electron loss $\varrho_{\mathrm{M}}$ of $\mathrm{Al}$ atom as 1.74 . Using a value of 0.25 for $\varepsilon$, they obtained values of 0.46 for $A_{\mathrm{M}}$ and 0.17 for $B_{\mathrm{M}}$. Since (15) is a sum of the contributions from two terminal and two bridging bonds to the EFG component along the direction connecting two bridging $\mathrm{Br}$ atoms, the contribution of one terminal bond to the EFG along the bond is given by

$$
\left(A_{\mathrm{M}} / 2\right)\left[1+\cot ^{2}\left(\phi_{12} / 2\right)\right]\left(1+\varrho_{\mathrm{M}} \varepsilon\right) /\left[3 \sin ^{2}\left(\phi_{34} / 2\right)-1\right],
$$

which is evaluated to be 0.51 . Similarly, the contribution of one bridging bond is given by

$$
\left(B_{\mathrm{M}} / 2\right)\left[1+\cot ^{2}\left(\phi_{12} / 2\right)\right]\left(1+\varrho_{\mathrm{M}} \varepsilon\right),
$$

and this is evaluated to be 0.21 .

The values of $\lambda$ in Table 2 should be compared with the average of these values. In the preceding paper [1] the low value of $\lambda$ for $\mathrm{Br}$ atoms was attributed to the low estimate of $\theta_{\mathrm{D}}$. For $\mathrm{Al}$ atom, certainly, corresponding to the much higher $\theta_{\mathrm{D}}$, a much larger $\lambda$ has been obtained. However, the values of $\lambda$ are still smaller than either value of $f$ for each bond. Since in (12) and (13) the first terms are positive and dominant, we may expect that these equations approximately give the lower and upper limits of the observed transi- tion probability $W_{\mathrm{obs}}$, respectively. However, the present situation is expressed in terms of $W$ as

$$
\begin{aligned}
W_{\text {obs }} & <W_{\text {calc }} \text { (bridging neglected) } \\
& <W_{\text {calc }}(\text { bridging included }),
\end{aligned}
$$

whichever value of $f$ for each bond is used for $\lambda$.

\subsection{Concluding Remarks}

By the use of NMR the problem originating from the mixing of spin states in the NQR has been avoided. An approach to the quadrupolar relaxation in a multi-bond system by means of the theory of the Raman process has been shown. The estimated covalency is relatively small compared with the values estimated from the NQR frequencies. Nevertheless, taking into account the crudeness involved in the theory and the present analysis, it should still be noted that the theory of the Raman process gives a substantially correct order of magnitude of covalency.

\section{Appendix}

\section{Derivation of $N_{\mu v}$}

In (4.35) and (4.36) of [2],

$$
N_{\mu v}=\Sigma_{n, n^{\prime}} \Sigma_{p p^{\prime}} W_{\mu n}^{*}\left(\sigma \sigma^{\prime}\right) \mathrm{W}_{\mu n^{\prime}}\left(\sigma \sigma^{\prime}\right)
$$

is expressed with the direction cosines of bonds $\boldsymbol{n}$ and $\boldsymbol{n}^{\prime}$ in the coordinate system whose $z$ axis is taken along the external magnetic field $\boldsymbol{H}_{0}$. Due to the nature of the direction cosines, $N_{\mu \nu}$ can be written as a sum of terms consisting of the powers only of the direction cosines to the $z$ axis, $\gamma_{n}$ and $\gamma_{n^{\prime}}$. To average the powers

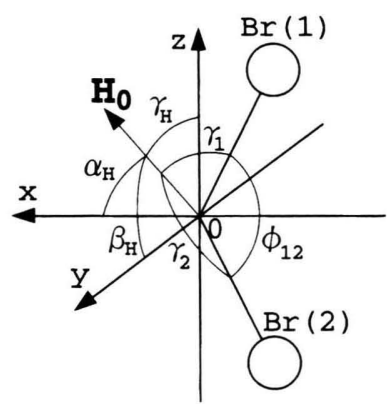

Fig. 5. The coordinate system for the calculation of the average of $N_{\mu v}$ with respect to the direction of the field $\boldsymbol{H}_{0}$. The Al atom is taken as the origin. The symbols $\gamma_{1}, \gamma_{2}, \alpha_{H}, \beta_{H}$, and $\gamma_{H}$ denote the direction cosines, while $\phi_{12}$ denotes the angle. 
of, say $\gamma_{1}$ and $\gamma_{2}$ with respect to the direction of $\boldsymbol{H}_{0}$, we introduce the new coordinate system shown in Fig. 5, where the $-x$ axis is taken as the bisector of the bond angle $\mathrm{Br}(1)-\mathrm{Al}-\mathrm{Br}(2)$, and the $z$ axis is taken in the plane of these bonds. Then $\gamma_{1}$ is written as the inner product of the unit vector along the bond $\mathrm{Al}-\mathrm{Br}(1),\left(-\cos \left(\phi_{12} / 2\right), 0, \sin \left(\phi_{12} / 2\right)\right)$, and the unit vector along the field, $\left(\alpha_{H}, \beta_{H}, \gamma_{H}\right)$, as

$$
\gamma_{1}=-\alpha_{H} \cos \left(\phi_{12} / 2\right)+\gamma_{H} \sin \left(\phi_{12} / 2\right) \text {. }
$$

Similarly,

$$
\gamma_{2}=-\alpha_{H} \cos \left(\phi_{12} / 2\right)-\gamma_{H} \sin \left(\phi_{12} / 2\right) .
$$

Then we get

$$
\begin{aligned}
& \overline{\gamma_{1}^{2}}=\overline{\gamma_{2}^{2}}=1 / 3, \\
& \overline{\gamma_{1}^{4}}=\overline{\gamma_{2}^{4}}=1 / 5,
\end{aligned}
$$

[1] N. Okubo, M. Igarashi, R. Yoshizaki, Z. Naturforsch. 50 a, 737 (1995).

[2] K. Yosida and T. Moriya, J. Phys. Soc. Japan 11, 33 (1956).

[3] R. G. Barnes and S. L. Segel, J. Chem. Phys. 25, 180 (1956).

[4] P. A. Casabella, P. J. Bray, and R. G. Barnes, J. Chem. Phys. 30, 1393 (1950)

[5] T. Okuda, H. Terao, O. Ege, and H. Negita, J. Chem. Phys. 52, 5489 (1970).

[6] N. Weiden and A. Weiss, J. Magn. Resn. 20, 334 (1975).

[7] H. Zabel and S. A. Solin eds., Graphite Intercalation Compounds I, Springer, Berlin 1990.

$$
\begin{aligned}
& \overline{\gamma_{1} \gamma_{2}}=(1 / 3) \cos \phi_{12}, \\
& \overline{\gamma_{1}^{2} \gamma_{2}^{2}}=(1 / 15)\left(2 \cos ^{2} \phi_{12}+1\right), \\
& \overline{\gamma_{1}^{3} \gamma_{2}}=\overline{\gamma_{1} \gamma_{2}^{3}}=(1 / 5) \cos \phi_{12} .
\end{aligned}
$$

Here the averages

$$
\overline{\alpha_{H}^{2}}=\overline{\gamma_{H}^{2}}=1 / 3, \quad \overline{\alpha_{H}^{4}}=\overline{\gamma_{H}^{4}}=1 / 5, \quad \overline{\alpha_{H}^{2} \gamma_{H}^{2}}=1 / 15,
$$

and

$$
\overline{\alpha_{H}^{3} \gamma_{H}}=\overline{\alpha_{H} \gamma_{H}^{3}}=0
$$

have been used. Since the coordinate axes have been chosen irrespectively of the principal axis system, the above result can be generalized to any bond pair. Substitution of the results into the direction averages in $\Sigma_{p p^{\prime}} W_{\mu n}^{*}\left(\sigma \sigma^{\prime}\right) W_{\mu n^{\prime}}\left(\sigma \sigma^{\prime}\right)$ yields the expressions for $N_{\mu v}$ in the text.

[8] To be published elsewhere.

[9] G. H. Stauss, J. Chem. Phys. 40, 1988 (1964).

[10] E. R. Andrew and D. P. Tunstall, Proc. Phys. Soc. 78, 1 (1961).

[11] P. A. Renes and C. H. Mac Gillavry, Rec. Trav. Chim. 64, 275 (1945).

[12] E. A. C. Lucken, Nuclear Quadrupole Coupling Constants, Academic Press, New York 1969.

[13] N. Okubo and Y. Abe, Z. Naturforsch. 49a, 680 (1994).

[14] T. P. Das and E. L. Hahn, Nuclear Quadrupole Resonance Spectroscopy, Solid State Physics, F. Seitz and D. Turnbull, Academic Press, New York 1958, Supplement (1994). 